\title{
Preliminary design of a bunching system for the CLIC polarized electron source
}

\author{
F. Zhou, A. Brachmann, and J. Sheppard (SLAC) \\ SLAC National Accelerator Laboratory, Menlo Park, CA 94025
}

\section{Introduction}

Major parameters of the CLIC and ILC electron sources are given in Table I. It is shown that the CLIC source needs to provide 312 15-ps-long 2-GHz microbunches. There are two approaches to achieve the time structure [2]: one is to develop a 2-GHz optical pulse train, and the other to develop a 156-ns-long CW optical pulse and use an RF bunching system to generate 312 2-GHz microbunches. The former scheme may ease the RF bunching system but still need it to bunch 100-ps of microbunch down to 15-ps level. Otherwise, a huge amount of energy spread is accumulated when the beam is accelerated through downstream 2-GHz accelerator. In addition, in the former scheme, the space charge is high and surface charge is not yet proven in the parameter regime and 2-GHz mode locked laser is challenging. The latter scheme needs a high-efficiency bunching system to generate 312 15-ps microbunches with 2-GHz repetition rate but it has some notable advantages: a 156-ns CW laser technique is matured, and the charge limit behavior in the scheme is better characterized than that in the former case, as listed in the table. This note presents a design and modeling of the bunching system for the latter scheme to convert a 156-ns CW pulse to 312 15-ps long 2-GHz microbunches.

Table I: Major parameters of the ILC and CLIC electron sources

\begin{tabular}{|l|c|c|c|}
\hline E-source parameters & ILC & $\begin{array}{c}\text { CLIC } \\
\text { (original) [1] }\end{array}$ & $\begin{array}{c}\text { CLIC } \\
\text { (SLAC proposed) }\end{array}$ \\
\hline Number of microbunches @cathode & 2625 & 312 & 1 DC beam \\
\hline Electrons/(micro)bunch @cathode & $5 \mathrm{nC}$ & $0.96 \mathrm{nC}$ & $300 \mathrm{nC}$ \\
\hline Number of microbunches @injector & 2625 & 312 & 312 \\
\hline Width of (micro)bunch @cathode & $1.3 \mathrm{~ns}$ & $\sim 100 \mathrm{ps}$ & $156 \mathrm{~ns} \mathrm{DC}$ \\
\hline Width of microbunch @injector & $20 \mathrm{ps}$ & - & $14 \mathrm{ps}$ \\
\hline Micropulse repetition rate @cathode & $3 \mathrm{MHz}$ & $2-\mathrm{GHz}$ & - \\
\hline Microbunch repetition rate @injector & $3 \mathrm{MHz}$ & $2-\mathrm{GHz}$ & $2-\mathrm{GHz}$ \\
\hline Width of Macropulse & $1 \mathrm{~ms}$ & $156 \mathrm{~ns}$ & $156 \mathrm{~ns}$ \\
\hline Macropulse repetition rate & $5 \mathrm{~Hz}$ & $50 \mathrm{~Hz}$ & $50 \mathrm{~Hz}$ \\
\hline Charge per macropulse & $13125 \mathrm{nC}$ & $300 \mathrm{nC}$ & $300 \mathrm{nC}$ \\
\hline Average current from gun & $66 \mu \mathrm{A}$ & $15 \mu \mathrm{A}$ & $15 \mu \mathrm{A}$ \\
\hline Peak current @cathode & $4.0 \mathrm{~A}$ & $9.6 \mathrm{~A}$ & $1.9 \mathrm{~A}$ \\
\hline Current intensity @1cm radius & $1.25 \mathrm{~A} / \mathrm{cm}^{2}$ & $3.0 \mathrm{~A} / \mathrm{cm}^{2}$ & $0.64 \mathrm{~A} / \mathrm{cm}^{2}$ \\
\hline Polarization & $>80 \%$ & $>80 \%$ & $>80 \%$ \\
\hline
\end{tabular}




\section{Bunching system}

A bunching system comprising two 2-GHz prebunchers, one 5-cell tapered- $\beta$ buncher, and 2-GHz accelerator is proposed, as shown in Figure 1. While two prebunchers are used to modulate macrobunch, a 5-cell tapered- $\beta$ travelling wave $2-\mathrm{GHz}$ structures are used as a buncher to compress the microbunches down to 14-ps FWHM. Then 2-GHz accelerator downstream of the buncher accelerates the beam to 19-MeV. Higher energy can be easily achieved by adding more RF structures but the bunching performance will not be changed. Distance from the gun to the first prebuncher and from the first to the second prebuncher is $56.5-\mathrm{cm}$ and $54-\mathrm{cm}$, respectively. $14-\mathrm{kV}$ and $36-\mathrm{kV}$ of voltages are applied into the first and second prebunchers respectively to modulate the 156-ns pulse. The beam transported from the gun to the first pre-buncher is focused by two magnetic lenses so that they can be used to adjust the radius and convergence of the beam at the entrance to the solenoid that confines the beam in the following second pre-buncher and buncher. While primary bunching is achieved in a 5-cell 2-GHz traveling wave tapered- $\beta$ from 0.75 to $0.93 \mathrm{RF}$ structure running at the gradient of $\sim 8.6 \mathrm{MV} / \mathrm{m}$, a final, small increment of bunching takes place in the first several cells in the 2-GHz accelerator, which immediately follows the buncher. $14 \mathrm{MV} / \mathrm{m}$ of the accelerating gradient in the structure is used in the simulations. The first several cells of the 2-GHz buncher and accelerator are immersed in a 1700-G solenoid field to focus the beam, and then the field is tapered down to zero as the beam gains energy. The field map along the beamline from the gun to the accelerator is shown in Figure 2. The generation of 2-GHz microbunches and its beam dynamics are modeled with PARMELA. In principle, the generation of 312 2-GHz microbunches from 156-ns DC beam (i.e., 312 2-GHz RF periods) transporting through the bunching system can be simulated. To simplify the simulations, initial pulse duration of four 2-GHz RF periods is modeled as an example to generate four microbunches with the bunching system. The initial pulse duration and final pulse structure at injector exit are shown in Figs. 3a and 3b, respectively. The final microbunch and its energy spectrum at $19-\mathrm{MeV}$ are shown in Figs. 4a and 4b, respectively. 88\% of electrons from the gun are captured within a window of $\Delta \mathrm{t} \times \Delta \mathrm{E}=30 \mathrm{ps} \times 0.45 \mathrm{MeV}$ at 19$\mathrm{MeV}$. The simulation results are summarized in Table II.

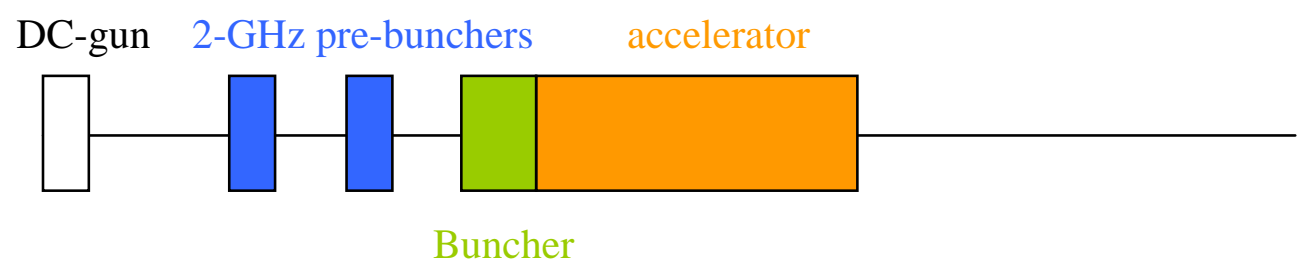

Figure 1: The schematic layout of bunching system for CLIC electron source. 


\section{Conclusion and outlook}

A bunching system to generate a train of microbunches with 2-GHz repetition rate from a macropulse for the CLIC injector is preliminarily designed and modeled. The tracking shows that $88 \%$ of electrons from the DC-gun are captured within a window of $30 \mathrm{ps} \times 0.45 \mathrm{MeV}$ at $19-\mathrm{MeV}$. Looking toward the technical design, more detailed work is needed including: (1) adding more RF structures to get energy at about $80 \mathrm{MeV}$; (2) bunching system optimizations to meet the engineering design; (3) detail definitions of system components.

\section{References}

[1] L. Rinolfi, CLIC workshop, 2007.

[2] J. Sheppard, A. Brachmann, and F. Zhou, Proposal to demonstrate a polarized electron source for CLIC, unpublished, 2009.

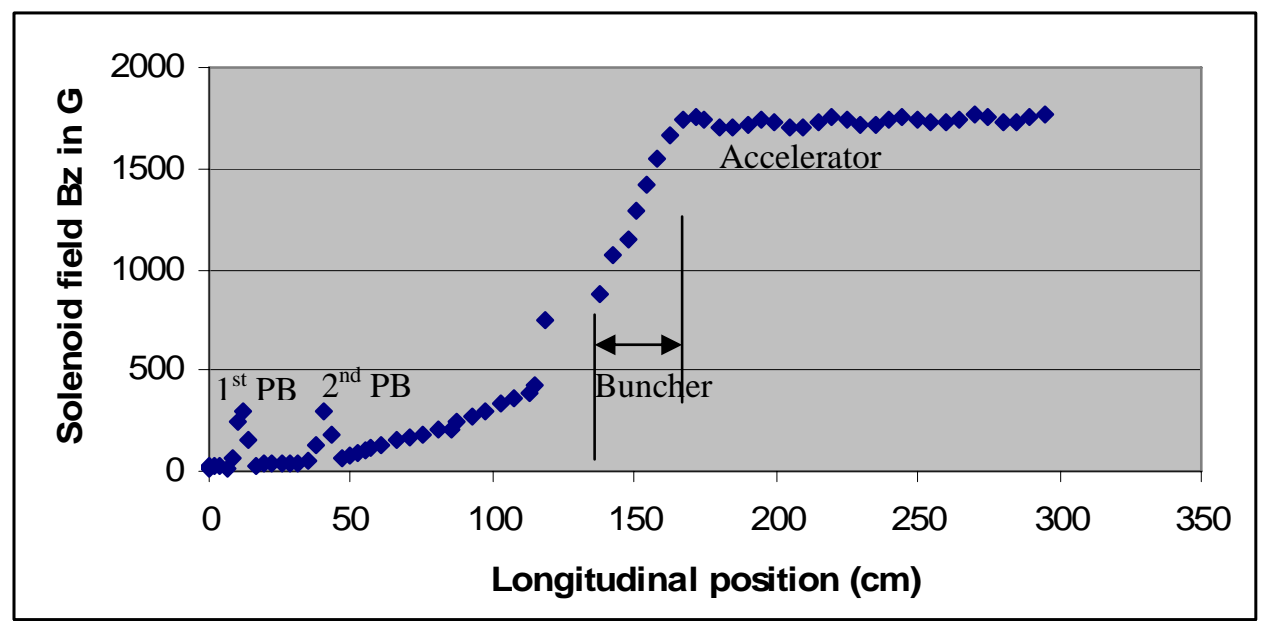

Figure 2: Field map along the beamline. PB is prebuncher.

Table II: Summary of modeling results

\begin{tabular}{|c|c|}
\hline $\begin{array}{l}\text { Gun voltage } \\
\text { Injector energy }\end{array}$ & $\begin{array}{l}140 \mathrm{kV} \\
19 \mathrm{MeV}\end{array}$ \\
\hline $\begin{array}{l}\text { Charge required/microbunch @inj } \\
\text { Efficiency from gun to injector exit } \\
\text { Achieved charge/microbunch within a } \\
\text { window }(\Delta \mathrm{t} \times \Delta \mathrm{E}=30 \mathrm{ps} \times 0.45 \mathrm{MeV})\end{array}$ & $\begin{array}{l}\sim 1 \mathrm{nC} \\
88 \% \\
1.3 \mathrm{nC}\end{array}$ \\
\hline $\begin{array}{l}\text { Initial DC pulse length on cathode } \\
\text { Final phase extension FWHM (FW) } \\
\text { \# of generated microbunches @ inj }\end{array}$ & $\begin{array}{l}156 \mathrm{~ns} \\
14 \mathrm{ps}(30 \mathrm{ps}) \\
312\end{array}$ \\
\hline Final energy spread FWHM (FW) & $100 \mathrm{keV}(1 \mathrm{MeV})$ \\
\hline Norm. rms emittance at injector exit & $22 \mu \mathrm{m}$ \\
\hline
\end{tabular}



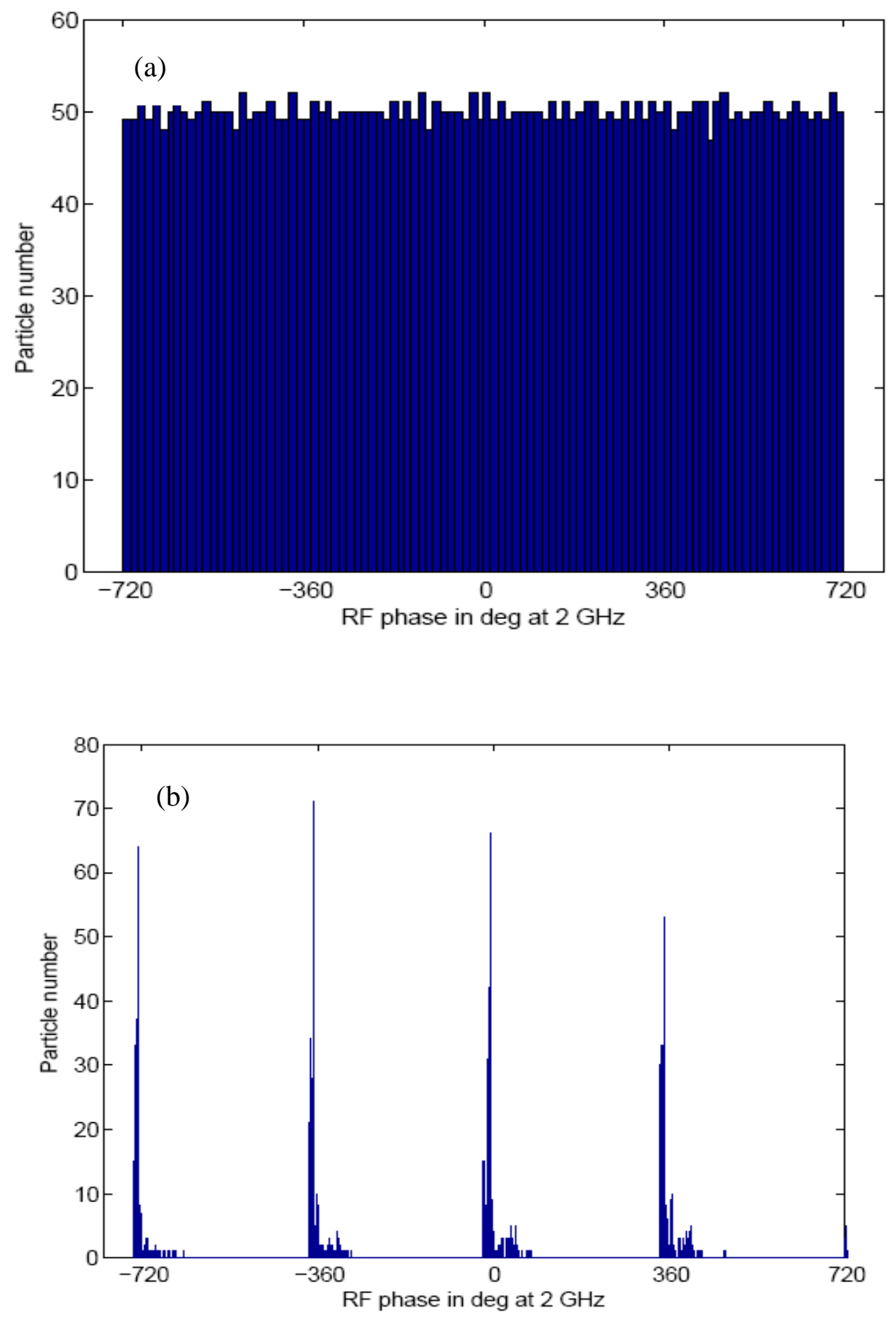

Figure 3: Initial pulse duration (a) on the cathode, and final bunched pulse structure (b) at $19 \mathrm{MeV}$ 

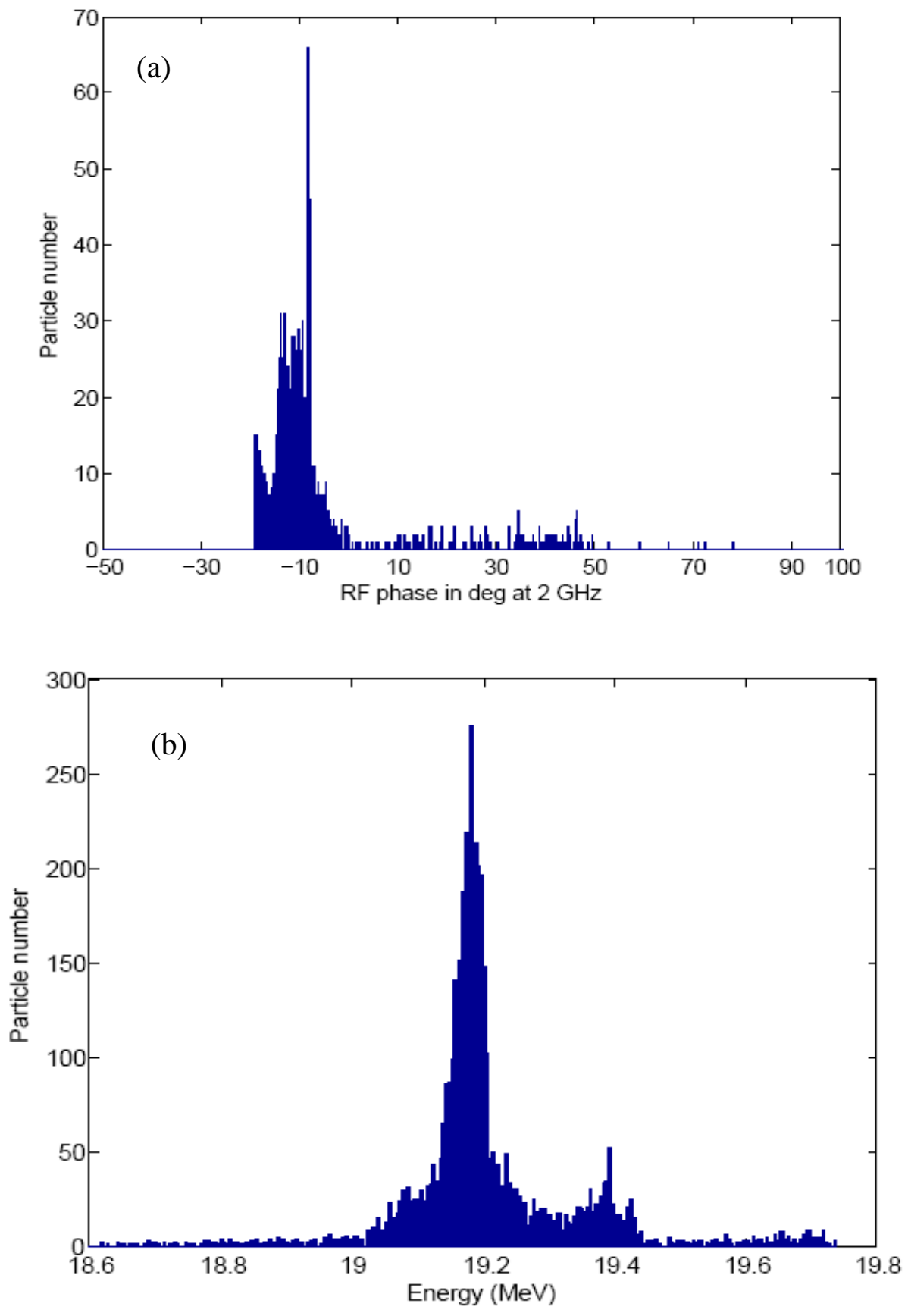

Figure 4: Microbunch (a) and energy spectrum (b) at $19 \mathrm{MeV}$ 